\title{
On the second Dirichlet eigenvalue of some nonlinear anisotropic elliptic operators
}

\author{
Francesco Della Pietra, Nunzia Gavitone, Gianpaolo Piscitelli \\ Università degli studi di Napoli Federico II, Dipartimento di Matematica e Applicazioni "R. Caccioppoli" \\ Via Cintia, Monte S. Angelo - 80126 Napoli, Italia.*
}

October 22, 2018

Let $\Omega$ be a bounded open set of $\mathbb{R}^{n}, n \geqslant 2$. In this paper we mainly study some properties of the second Dirichlet eigenvalue $\lambda_{2}(p, \Omega)$ of the anisotropic $p$ Laplacian

$$
-Q_{\mathfrak{p}} u:=-\operatorname{div}\left(\mathrm{F}^{\mathrm{p}-1}(\nabla u) \mathrm{F}_{\xi}(\nabla u)\right),
$$

where $F$ is a suitable smooth norm of $\mathbb{R}^{n}$ and $\left.p \in\right] 1,+\infty[$. We provide a lower bound of $\lambda_{2}(p, \Omega)$ among bounded open sets of given measure, showing the validity of a Hong-Krahn-Szego type inequality. Furthermore, we investigate the limit problem as $p \rightarrow+\infty$.

MSC 2010: $35 \mathrm{P}_{15}-35 \mathrm{P}_{30}-35 \mathrm{~J} 60$

Keywords: Nonlinear eigenvalue problems - Hong-Krahn-Szego inequality - Finsler metrics

\section{INTRODUCTION}

Let $\Omega$ be a bounded open set of $\mathbb{R}^{n}, n \geqslant 2$. The main aim of this paper is to study some properties of the Dirichlet eigenvalues of the anisotropic $p$-Laplacian operator:

$$
-Q_{\mathfrak{p}} u:=-\operatorname{div}\left(\mathrm{F}^{\mathrm{p}-1}(\nabla \mathrm{u}) \nabla_{\xi} \mathrm{F}(\nabla \mathrm{u})\right),
$$

where $1<p<+\infty$, and $F$ is a sufficiently smooth norm on $\mathbb{R}^{n}$ (see Section 2 for the precise assumptions on $\mathrm{F}$ ), namely the values $\lambda$ such that the problem

$$
\begin{cases}-Q_{p} u=\lambda|u|^{p-2} u & \text { in } \Omega \\ u=0 & \text { on } \partial \Omega\end{cases}
$$

admits a nontrivial solution in $W_{0}^{1, p}(\Omega)$.

The operator in (1) reduces to the $p$-Laplacian when $F$ is the Euclidean norm on $\mathbb{R}^{n}$. For a general norm $F, Q_{\mathfrak{p}}$ is anisotropic and can be highly nonlinear. In literature, several papers

\footnotetext{
*Email: f.dellapietra@unina.it, nunzia.gavitone@unina.it, gianpaolo.piscitelli@unina.it
} 
are devoted to the study of the smallest eigenvalue of (2), denoted by $\lambda_{1}(p, \Omega)$, in bounded domains (see Section 2), which has the variational characterization

$$
\lambda_{1}(p, \Omega)=\min _{\varphi \in W_{0}^{1, p}(\Omega) \backslash\{0\}} \frac{\int_{\Omega} F^{p}(\nabla \varphi) d x}{\int_{\Omega}|\varphi|^{p} d x} .
$$

Let $\Omega$ be a bounded domain. It is known (see [BFK, DG2]) that $\lambda_{1}(p, \Omega)$ is simple, the eigenfunctions have constant sign and it is isolated; moreover the only positive eigenfunctions are the first eigenfunctions. Furthermore, the Faber-Krahn inequality holds (see [BFK]):

$$
\lambda_{1}(p, \Omega) \geqslant \lambda_{1}\left(p, \mathcal{W}_{R}\right)
$$

where $\mathcal{W}_{R}$ is the so-called Wulff shape, that is the ball with respect to the dual norm Fo of $F$, having the same measure of $\Omega$ (see Section 2). Many other results are known for $\lambda_{1}(p, \Omega)$. The interested reader may refer, for example, to [BFK, BKJ, BGM, DG3, KN, P, WX]. As matter of fact, also different kind of boundary conditions have been considered as, for example, in the papers [DG, DGP] (Neumann case), [DG2] (Robin case).

Among the results contained in the quoted papers, we recall that if $\Omega$ is a bounded domain, it has been proved in $[\mathrm{BKJ}]$ that

$$
\lim _{p \rightarrow \infty} \lambda_{1}(p, \Omega)^{\frac{1}{p}}=\frac{1}{\rho_{F}(\Omega)},
$$

where $\rho_{F}(\Omega)$ is the anisotropic inradius of $\Omega$ with respect to the dual norm (see Section 2 for its definition), generalizing a well-known result in the Euclidean case contained in [JLM].

Actually, very few results are known for higher eigenvalues in the anisotropic case. In [F] the existence of a infinite sequence of eigenvalues is proved, obtained by means of a min - max characterization. Actually, as in the Euclidean case, it is not known if this sequence exhausts all the set of the eigenvalues. Here we will show that the spectrum of $-Q_{p}$ is a closed set, that the eigenfunctions are in $C^{1, \alpha}(\Omega)$ and that admit a finite number of nodal domains. We recall the reference [L2], where many results for the spectrum of the p-Laplacian in the Euclidean case have been summarized.

The core of the paper relies in the study of the second eigenvalue $\left.\lambda_{2}(p, \Omega), p \in\right] 1,+\infty[$, in bounded open sets, defined as

$$
\lambda_{2}(p, \Omega):= \begin{cases}\min \left\{\lambda>\lambda_{1}(p, \Omega): \lambda \text { is an eigenvalue }\right\} & \text { if } \lambda_{1}(p, \Omega) \text { is simple } \\ \lambda_{1}(p, \Omega) & \text { otherwise, }\end{cases}
$$

and in analyzing its behavior when $p \rightarrow \infty$.

First of all, we show that if $\Omega$ is a domain, then $\lambda_{2}(p, \Omega)$ admits exactly two nodal domains. Moreover, for a bounded open set $\Omega$, we prove a sharp lower bound for $\lambda_{2}$, namely the Hong-Krahn-Szego inequality

$$
\lambda_{2}(p, \Omega) \geqslant \lambda_{2}(p, \widetilde{\mathcal{W}}),
$$

where $\widetilde{\mathcal{W}}$ is the union of two disjoint Wulff shapes, each one of measure $\frac{|\Omega|}{2}$.

In the Euclidean case, such inequality is well-known for $p=2$, and it has been recently studied for any $1<p<+\infty$ in [BF2].

Finally, we address our attention to the behavior of $\lambda_{2}(p, \Omega)$ when $\Omega$ is a bounded open set and $p \rightarrow+\infty$. In particular, we show that

$$
\lim _{p \rightarrow \infty} \lambda_{2}(p, \Omega)^{\frac{1}{p}}=\frac{1}{\rho_{2, F}(\Omega)},
$$


where $\rho_{2, \mathrm{~F}}(\Omega)$ is the radius of two disjoint Wulff shapes $\mathcal{W}_{1}, \mathcal{W}_{2}$ such that $\mathcal{W}_{1} \cup \mathcal{W}_{2}$ is contained in $\Omega$. Furthermore, the normalized eigenfunctions of $\lambda_{p}(2, \Omega)$ converge to a function $u_{\infty}$ that is a viscosity solution to a suitable fully nonlinear elliptic problem (see Section 5 ). In the Euclidean case, this kind of result has been proved for bounded domains in [JL]. We consider both the nonconnected case and general norm F. In a forthcoming paper we will deal with the limit case $p \rightarrow 1$.

As enhanced before, the aim of the paper is twofold: first, to consider the case of a general Finsler norm F; second, to extend also the results known in the case of domains, to the case of nonconnected sets. We structured the paper as follows. In Section 2 we recall the main definitions as well as some basic fact of convex geometry, and we fix the precise assumptions on $F$. In Section 3 we state the general eigenvalue problem for $-Q_{p}$, recalling some known results, extending them, where it is possible, to the case of nonconnected sets; moreover we provide several properties of the first and of higher eigenvalues and eigenfunctions. In Section 4 the attention will be focused on the second eigenvalue $\lambda_{2}(p, \Omega)$ of $-Q_{p}$ proving, among the other properties of $\lambda_{2}$, the Hong-Krahn-Szego inequality. Finally, in Section 5 we study the limit case $p \rightarrow \infty$.

\section{NOTATION AND PRELIMINARIES}

Throughout the paper we will consider a convex even 1-homogeneous function

$$
\xi \in \mathbb{R}^{\mathrm{n}} \mapsto \mathrm{F}(\xi) \in[0,+\infty[,
$$

that is a convex function such that

$$
F(t \xi)=|t| F(\xi), \quad t \in \mathbb{R}, \xi \in \mathbb{R}^{n},
$$

and such that

$$
a|\xi| \leqslant F(\xi), \quad \xi \in \mathbb{R}^{n},
$$

for some constant $0<a$. Under this hypothesis it is easy to see that there exists $b \geqslant a$ such that

$$
\mathrm{F}(\xi) \leqslant \mathrm{b}|\xi|, \quad \xi \in \mathbb{R}^{\mathrm{n}} .
$$

Moreover, throughout the paper we will assume that

$$
\nabla_{\xi}^{2}\left[F^{p}\right](\xi) \text { is positive definite in } \mathbb{R}^{\mathfrak{n}} \backslash\{0\},
$$

with $1<p<+\infty$.

The hypothesis (6) on F assures that the operator

$$
\mathcal{Q}_{\mathrm{p}}[\mathrm{u}]:=\operatorname{div}\left(\frac{1}{\mathrm{p}} \nabla_{\xi}\left[\mathrm{F}^{\mathrm{p}}\right](\nabla \mathrm{u})\right)
$$

is elliptic, hence there exists a positive constant $\gamma$ such that

$$
\frac{1}{p} \sum_{i, j=1}^{n} \nabla_{\xi_{i} \xi_{j}}^{2}\left[F^{p}\right](\eta) \xi_{i} \xi_{j} \geqslant \gamma|\eta|^{p-2}|\xi|^{2},
$$

for some positive constant $\gamma$, for any $\eta \in \mathbb{R}^{\mathfrak{n}} \backslash\{0\}$ and for any $\xi \in \mathbb{R}^{\mathfrak{n}}$. 
Remark 2.1. We stress that for $p \geqslant 2$ the condition $\nabla_{\xi}^{2}\left[F^{2}\right](\xi)$ is positive definite in $\mathbb{R}^{n} \backslash\{0\}$,

implies (6).

The polar function $\mathrm{F}^{\mathrm{o}}: \mathbb{R}^{\mathrm{n}} \rightarrow[0,+\infty[$ of $\mathrm{F}$ is defined as

$$
\mathrm{F}^{\mathrm{o}}(v)=\sup _{\xi \neq 0} \frac{\langle\xi, v\rangle}{\mathrm{F}(\xi)} .
$$

It is easy to verify that also $F^{\circ}$ is a convex function which satisfies properties (4) and (5). Furthermore,

$$
\mathrm{F}(v)=\sup _{\xi \neq 0} \frac{\langle\xi, v\rangle}{\mathrm{Fo}^{\circ}(\xi)}
$$

From the above property it holds that

$$
|\langle\xi, \eta\rangle| \leqslant F(\xi) F^{o}(\eta), \quad \forall \xi, \eta \in \mathbb{R}^{n} .
$$

The set

$$
\mathcal{W}=\left\{\xi \in \mathbb{R}^{n}: F^{o}(\xi)<1\right\}
$$

is the so-called Wulff shape centered at the origin. We put $\kappa_{n}=|\mathcal{W}|$, where $|\mathcal{W}|$ denotes the Lebesgue measure of $\mathcal{W}$. More generally, we denote with $\mathcal{W}_{r}\left(x_{0}\right)$ the set $r \mathcal{W}+x_{0}$, that is the Wulff shape centered at $x_{0}$ with measure $\kappa_{n} r^{n}$, and $\mathcal{W}_{r}(0)=\mathcal{W}_{r}$.

The following properties of $F$ and $F^{\circ}$ hold true (see for example [BP]):

$$
\begin{aligned}
& \left\langle\nabla_{\xi} \mathrm{F}(\xi), \xi\right\rangle=\mathrm{F}(\xi), \quad\left\langle\nabla_{\xi} \mathrm{F}^{\mathrm{o}}(\xi), \xi\right\rangle=\mathrm{F}^{\mathrm{o}}(\xi), \\
& \mathrm{F}\left(\nabla_{\xi} \mathrm{F}^{\mathrm{o}}(\xi)\right)=\mathrm{F}^{\mathrm{o}}\left(\nabla_{\xi} \mathrm{F}(\xi)\right)=1, \quad \forall \xi \in \mathbb{R}^{\mathrm{n}} \backslash\{0\}, \\
& \mathrm{F}^{\mathrm{o}}(\xi) \nabla_{\xi} \mathrm{F}\left(\nabla_{\xi} \mathrm{F}^{\mathrm{o}}(\xi)\right)=\mathrm{F}(\xi) \nabla_{\xi} \mathrm{F}^{\mathrm{o}}\left(\nabla_{\xi} \mathrm{F}(\xi)\right)=\xi, \quad \forall \xi \in \mathbb{R}^{\mathrm{n}} \backslash\{0\} .
\end{aligned}
$$

Finally, we will recall the following

Definition 1. A domain of $\mathbb{R}^{n}$ is a connected open set.

\section{THE DIRICHLET EIGENVALUE PROBLEM FOR $-Q_{p}$}

Here we state the eigenvalue problem for $Q_{p}$. Let $\Omega$ be a bounded open set in $\mathbb{R}^{n}, n \geqslant 2$, $1<\mathrm{p}<+\infty$, and consider the problem

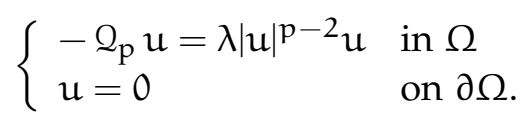

Definition 2. We say that $u \in W_{0}^{1, p}(\Omega), u \neq 0$, is an eigenfunction of $(7)$, if

$$
\int_{\Omega}\left\langle\mathrm{F}^{\mathrm{p}-1}(\nabla \mathrm{u}) \nabla_{\xi} \mathrm{F}(\nabla \mathrm{u}), \nabla \varphi\right\rangle \mathrm{d} x=\lambda \int_{\Omega}|\mathrm{u}|^{\mathrm{p}-2} \mathrm{u} \varphi \mathrm{d} x
$$

for all $\varphi \in W_{0}^{1, p}(\Omega)$. The corresponding real number $\lambda$ is called an eigenvalue of (7).

Obviously, if $u$ is an eigenfunction associated to $\lambda$, then

$$
\lambda=\frac{\int_{\Omega} F^{p}(\nabla u) d x}{\int_{\Omega}|u|^{p} d x}>0 .
$$


3.1 The first eigenvalue

Among the eigenvalues of (7), the smallest one, denoted here by $\lambda_{1}(p, \Omega)$, has the following well-known variational characterization:

$$
\lambda_{1}(p, \Omega)=\min _{\varphi \in W_{0}^{1, p}(\Omega) \backslash\{0\}} \frac{\int_{\Omega} F^{p}(\nabla \varphi) d x}{\int_{\Omega}|\varphi|^{p} d x} .
$$

In the following theorems its main properties are recalled.

Theorem 3.1. If $\Omega$ is a bounded open set in $\mathbb{R}^{n}, n \geqslant 2$, there exists a function $u_{1} \in C^{1, \alpha}(\Omega) \cap C(\bar{\Omega})$ which achieves the minimum in (9), and satisfies the problem (7) with $\lambda=\lambda_{1}(p, \Omega)$. Moreover, if $\Omega$ is connected, then $\lambda_{1}(\mathrm{p}, \Omega)$ is simple, that is the corresponding eigenfunctions are unique up to a multiplicative constant, and the first eigenfunctions have constant sign in $\Omega$.

Proof. The proof can be immediately adapted from the case of $\Omega$ connected and we refer the reader, for example, to [L, BFK].

Theorem 3.2. Let $\Omega$ be a bounded open set in $\mathbb{R}^{n}, n \geqslant 2$. Let $u \in W_{0}^{1, p}(\Omega)$ be an eigenfunction of (7) associated to an eigenvalue $\lambda$. If $u$ does not change sign in $\Omega$, then there exists a connected component $\Omega_{0}$ of $\Omega$ such that $\lambda=\lambda_{1}\left(p, \Omega_{0}\right)$ and $u$ is a first eigenfunction in $\Omega_{0}$. In particular, if $\Omega$ is connected then $\lambda=\lambda_{1}(p, \Omega)$ and a constant sign eigenfunction is a first eigenfunction.

Proof. If $\Omega$ is connected, a proof can be found in [L, DG2]. Otherwise, if $u \geqslant 0$ in $\Omega$ disconnected, by the maximum principle $u$ must be either positive or identically zero in each connected component of $\Omega$. Hence there exists a connected component $\Omega_{0}$ such that $u$ coincides in $\Omega_{0}$ with a positive eigenfunction relative to $\lambda$. By the previous case, $\lambda=\lambda_{1}\left(p, \Omega_{0}\right)$ and the proof is completed.

Here we list some other useful and interesting properties that can be proved in a similar way than the Euclidean case.

Proposition 3.3. Let $\Omega$ be a bounded open set in $\mathbb{R}^{n}, \mathrm{n} \geqslant 2$, the following properties hold.

1. For $\mathrm{t}>0$ it holds $\lambda_{1}(\mathrm{p}, \mathrm{t} \Omega)=\mathrm{t}^{-\mathrm{p}} \lambda_{1}(\mathrm{p}, \Omega)$.

2. If $\Omega_{1} \subseteq \Omega_{2} \subseteq \Omega$, then $\lambda_{1}\left(p, \Omega_{1}\right) \geqslant \lambda_{1}\left(p, \Omega_{2}\right)$.

3. For all $1<p<s<+\infty$ we have $p\left[\lambda_{1}(p, \Omega)\right]^{1 / p}<s\left[\lambda_{1}(s, \Omega)\right]^{1 / s}$.

Proof. The first two properties are immediate from (9). As regards the third property, the inequality derives from the Hölder inequality, similarly as in [LI]. Indeed, taking $\phi=|\psi|^{\frac{s}{p}-1} \psi$, $\psi \in W_{0}^{1, p}(\Omega) \cap L^{\infty}(\Omega), \psi \geqslant 0$, we have by (4) that

$$
\left[\lambda_{1}(p, \Omega)\right]^{\frac{1}{p}} \leqslant \frac{s}{p}\left(\frac{\int_{\Omega}|\psi|^{s-p} F^{p}(\nabla \psi) d x}{\int_{\Omega}|\psi|^{s} d x}\right)^{\frac{1}{p}} \leqslant \frac{s}{p}\left(\frac{\int_{\Omega} F^{s}(\nabla \psi) d x}{\int_{\Omega}|\psi|^{s} d x}\right)^{\frac{1}{s}}
$$

By minimizing with respect to $\psi$, we get the thesis.

In addition, the Faber-Krahn inequality for $\lambda_{1}(p, \Omega)$ holds. 
Theorem 3.4. Let $\Omega$ be a bounded open set in $\mathbb{R}^{n}, n \geqslant 2$, then

$$
|\Omega|^{\mathrm{p} / \mathrm{N}} \lambda_{1}(p, \Omega) \geqslant \kappa_{N}^{\mathrm{p} / \mathrm{N}} \lambda_{1}(\mathrm{p}, \mathcal{W}) .
$$

Moreover, equality sign in (10) holds if $\Omega$ is homothetic to the Wulff shape.

The proof of this inequality, contained in $[\mathrm{BFK}]$, is based on a symmetrization technique introduced in [AFLT] (see [ET, FV] for the equality cases).

Using the previous result we can prove the following property of $\lambda_{1}(p, \Omega)$.

Proposition 3.5. Let $\Omega$ be a bounded domain in $\mathbb{R}^{n}, n \geqslant 2$. The first eigenvalue of $(7), \lambda_{1}(p, \Omega)$, is isolated.

Proof. We argue similarly as in [L2]. For completeness we give the proof. For convenience we write $\lambda_{1}$ instead of $\lambda_{1}(p, \Omega)$. Let $\lambda_{k} \neq \lambda_{1}$ a sequence of eigenvalues such that

$$
\lim _{k \rightarrow+\infty} \lambda_{k}=\lambda_{1}
$$

Let $u_{k}$ be a normalized eigenfunction associated to $\lambda_{k}$ that is,

$$
\lambda_{k}=\int_{\Omega} F^{p}\left(\nabla u_{k}\right) d x \quad \text { and } \quad \int_{\Omega}\left|u_{k}\right|^{p} d x=1
$$

By (II), there exists a function $u \in W_{0}^{1, p}(\Omega)$ such that, up to a subsequence

$$
u_{k} \rightarrow \mathfrak{u} \quad \text { in } L^{p}(\Omega) \quad \nabla u_{k} \rightarrow \nabla u \text { weakly in } L^{p}(\Omega) .
$$

By the strong convergence of $u_{k}$ in $L^{p}(\Omega)$ and, recalling that $F$ is convex, by weak lower semicontinuity, it follows that

$$
\int_{\Omega}|\mathfrak{u}|^{p} \mathrm{dx}=1 \text { and } \int_{\Omega} \mathrm{F}^{\mathfrak{p}}(\nabla \mathfrak{u}) \mathrm{d} x \leqslant \lim _{\mathrm{k} \rightarrow \infty} \lambda_{\mathrm{k}}=\lambda_{1} .
$$

Hence, $u$ is a first eigenfunction. On the other hand, being $u_{k}$ not a first eigenfunction, by Theorem 3.2 it has to change sign. Hence, the sets $\Omega_{k}^{+}=\left\{u_{k}>0\right\}$ and $\Omega_{k}^{-}=\left\{u_{k}<0\right\}$ are nonempty and, as a consequence of the Faber-Krahn inequality and of Theorem 3.2, it follows that

$$
\lambda_{k}=\lambda_{1}\left(p, \Omega_{k}^{+}\right) \geqslant \frac{C_{n, F}}{\left|\Omega_{k}^{+}\right|^{\frac{p}{n}}}, \quad \lambda_{k}=\lambda_{1}\left(p, \Omega_{k}^{-}\right) \geqslant \frac{C_{n, F}}{\left|\Omega_{k}^{-}\right|^{\frac{p}{n}}} .
$$

This implies that both $\left|\Omega_{\mathrm{k}}^{+}\right|$and $\left|\Omega_{\mathrm{k}}^{-}\right|$cannot vanish as $\mathrm{k} \rightarrow+\infty$ and finally, that $\mathfrak{u}_{\mathrm{k}}$ converges to a function $u$ which changes sign in $\Omega$. This is in contradiction with the characterization of the first eigenfunctions, and the proof is completed.

\subsection{Higher eigenvalues}

First of all, we recall the following result (see [F, Theorem 1.4.1] and the references therein), which assures the existence of infinite eigenvalues of $-2_{p}$. We use the following notation. Let $\mathrm{S}^{\mathrm{n}-1}$ be the unit Euclidean sphere in $\mathbb{R}^{\mathrm{n}}$, and

$$
M=\left\{u \in W_{0}^{1, p}(\Omega): \int_{\Omega}|u|^{p} d x=1\right\}
$$

Moreover, let $\mathcal{C}_{n}$ be the class of all odd and continuous mappings from $\mathbb{S}^{n-1}$ to $M$. Then, for any fixed $f \in \mathcal{C}_{n}$, we have $f: \omega \in \mathbb{S}^{n-1} \mapsto f_{\omega} \in M$. 
Proposition 3.6. Let $\Omega$ a bounded open set of $\mathbb{R}^{n}$, for any $k \in \mathbb{N}$, the value

$$
\tilde{\lambda}_{k}(p, \Omega)=\inf _{f \in \mathfrak{e}_{n}} \max _{\omega \in S^{n-1}} \int_{\Omega} F^{p}\left(\nabla f_{\omega}\right) d x
$$

is an eigenvalue of $-Q_{p}$. Moreover,

$$
0<\tilde{\lambda}_{1}(p, \Omega)=\lambda_{1}(p, \Omega) \leqslant \tilde{\lambda}_{2}(p, \Omega) \leqslant \ldots \leqslant \tilde{\lambda}_{k}(p, \Omega) \leqslant \tilde{\lambda}_{k+1}(p, \Omega) \leqslant \ldots,
$$

and

$$
\tilde{\lambda}_{k}(p, \Omega) \rightarrow \infty \text { as } k \rightarrow \infty .
$$

Hence, we have at least a sequence of eigenvalues of $-Q_{p}$. Furthermore, the following proposition holds.

Proposition 3.7. Let $\Omega$ a bounded open set of $\mathbb{R}^{n}$. The spectrum of $-Q_{\mathrm{p}}$ is a closed set.

Proof. Let $\lambda_{k}$ be a sequence of eigenvalues converging to $\mu<+\infty$ and let $\mathfrak{u}_{k}$ be the corresponding normalized eigenfunctions, that is such that $\left\|\mathfrak{u}_{k}\right\|_{L^{p}(\Omega)}=1$. We have to show that $\mu$ is an eigenvalue of $-Q_{p}$.

We have that

$$
\int_{\Omega}\left\langle\mathrm{F}^{p-1}\left(\nabla \mathfrak{u}_{k}\right) \nabla_{\xi} \mathrm{F}\left(\nabla \mathfrak{u}_{k}\right), \nabla \varphi\right\rangle \mathrm{d} x=\lambda_{k} \int_{\Omega}\left|\mathfrak{u}_{k}\right|^{p-2} \mathfrak{u}_{k} \varphi \mathrm{d} x
$$

for any test function $\varphi \in W_{0}^{1, p}(\Omega)$. Since

$$
\lambda_{k}=\int_{\Omega} F^{p}\left(\nabla u_{k}\right) d x
$$

and being $\lambda_{k}$ a convergent sequence, up to a subsequence we have that there exists a function $u \in W_{0}^{1, p}(\Omega)$ such that $u_{k} \rightarrow u$ strongly in $L^{p}(\Omega)$ and $\nabla u_{k} \rightarrow \nabla u$ weakly in $L^{p}(\Omega)$. Our aim is to prove that $u$ is an eigenfunction relative to $\lambda$.

Choosing $\varphi=\mathfrak{u}_{k}-\mathfrak{u}$ as test function in the equation solved by $\mathfrak{u}_{k}$, we have

$$
\begin{aligned}
& \int_{\Omega}\left\langle\mathrm{F}^{p-1}\left(\nabla \mathfrak{u}_{k}\right) \nabla_{\xi} \mathrm{F}\left(\nabla \mathfrak{u}_{k}\right)-\mathrm{F}^{p-1}(\nabla \mathfrak{u}) \nabla_{\xi} \mathrm{F}(\nabla \mathfrak{u}), \nabla\left(\mathfrak{u}_{k}-\mathfrak{u}\right)\right\rangle \mathrm{d} x \\
& =\lambda_{k} \int_{\Omega}\left|\mathfrak{u}_{k}\right|^{p-2} \mathfrak{u}_{k}\left(\mathfrak{u}_{k}-\mathfrak{u}\right) \mathrm{d} x-\int_{\Omega} F^{p-1}(\nabla \mathfrak{u})\left\langle\nabla_{\xi} F(\nabla \mathfrak{u}), \nabla\left(\mathfrak{u}_{k}-\mathfrak{u}\right)\right\rangle \mathrm{d} x .
\end{aligned}
$$

By the strong convergence of $\mathfrak{u}_{k}$ and the weak one of $\nabla \mathfrak{u}_{k}$, the right-hand side of the above identity goes to zero as $k$ diverges. Hence

$$
\lim _{k \rightarrow \infty} \int_{\Omega}\left\langle\mathrm{F}^{\mathrm{p}-1}\left(\nabla \mathfrak{u}_{\mathrm{k}}\right) \nabla_{\xi} \mathrm{F}\left(\nabla \mathfrak{u}_{\mathrm{k}}\right)-\mathrm{F}^{\mathrm{p}-1}(\nabla \mathfrak{u}) \nabla_{\xi} \mathrm{F}(\nabla \mathfrak{u}), \nabla\left(\mathfrak{u}_{k}-\mathfrak{u}\right)\right\rangle \mathrm{d} x=0
$$

By nowadays standard arguments, this limit implies the strong convergence of the gradient, hence we can pass to the limit under the integral sign in (13) to obtain

$$
\int_{\Omega}\left\langle\mathrm{F}^{\mathrm{p}-1}(\nabla \mathrm{u}) \mathrm{F}_{\xi}(\nabla \mathrm{u}), \nabla \varphi\right\rangle \mathrm{d} x=\lambda \int_{\Omega}|\mathfrak{u}|^{\mathrm{p}-2} \mathrm{u} \varphi \mathrm{d} x
$$

This shows that $\lambda$ is an eigenvalue and the proof is completed.

Finally, we list some properties of the eigenfunctions, well-known in the Euclidean case (see for example [L2, AFT]). Recall that a nodal domain of an eigenfunction $u$ is a connected component of $\{u>0\}$ or $\{u<0\}$. 
Proposition 3.8. Let $\mathrm{p}>1$, and let $\Omega$ be a bounded open set in $\mathbb{R}^{n}$. Then the following facts hold.

(i) Any eigenfunction of $-Q_{\mathrm{p}}$ has only a finite number of nodal domains.

(ii) Let $\lambda$ be an eigenvalue of $-Q_{\mathrm{p}}$, and $\mathrm{u}$ be a corresponding eigenfunction. The following estimate holds:

$$
\|u\|_{L^{\infty}(\Omega)} \leqslant C_{n, p, F} \lambda^{\frac{\mathfrak{n}}{p}}\|u\|_{L^{1}(\Omega)}
$$

where $\mathrm{C}_{\mathrm{n}, \mathrm{p}, \mathrm{F}}$ is a constant depending only on $\mathrm{n}, \mathrm{p}$ and $\mathrm{F}$.

(iii) All the eigenfunctions of (1) are in $C^{1, \alpha}(\Omega)$, for some $\alpha \in(0,1)$.

Proof. Let $\lambda$ be an eigenvalue of $-Q_{p}$, and $u$ a corresponding eigenfunction.

In order to prove $(i)$, let us denote by $\Omega_{j}^{+}$a connected component of the set $\Omega^{+}:=\{u>0\}$. Being $\lambda=\lambda_{1}\left(\Omega_{j}^{+}\right)$, then by (10)

$$
\left|\Omega_{j}^{+}\right| \geqslant C_{n, p, F} \lambda^{-\frac{n}{p}} .
$$

Then, the thesis follows observing that

$$
|\Omega| \geqslant \sum_{j}\left|\Omega_{j}^{+}\right| \geqslant C_{n, p, F} \lambda^{-\frac{n}{p}} \sum_{j} 1 .
$$

In order to prove (ii), let $k>0$, and choose $\varphi(x)=\max \{u(x)-k, 0\}$ as test function in (8). Then

$$
\int_{A_{k}} F^{p}(\nabla u) d x=\lambda \int_{A_{k}}|u|^{p-2} u(u-k) d x
$$

where $A_{k}=\{x \in \Omega: \mathfrak{u}(x)>k\}$. Being $k\left|A_{k}\right| \leqslant\|u\|_{L^{1}(\Omega)}$, then $\left|A_{k}\right| \rightarrow 0$ as $k \rightarrow \infty$. By the inequality $a^{p-1} \leqslant 2^{p-1}(a-k)^{p-1}+2^{p-1} k^{p-1}$, we have

$$
\int_{A_{k}}|u|^{p-2} u(u-k) d x \leqslant 2^{p-1} \int_{A_{k}}(u-k)^{p} d x+2^{p-1} k^{p-1} \int_{A_{k}}(u-k) d x .
$$

By Poincaré inequality and property (5), then (15) and (16) give that

$$
\left(1-\lambda C_{n, p, F}\left|A_{k}\right|^{p / n}\right) \int_{A_{k}}(u-k)^{p} d x \leqslant \lambda\left|A_{k}\right|^{p / n} C_{n, p, F} k^{p-1} \int_{A_{k}}(u-k) d x .
$$

By choosing $k$ sufficiently large, the Hölder inequality implies

$$
\int_{A_{k}}(u-k) d x \leqslant \tilde{C}_{n, p, F} \lambda^{\frac{1}{p-1}} k\left|A_{k}\right|^{1+\frac{p}{n(p-1)}} .
$$

This estimate allows to apply [LU, Lemma 5.1, p. 71] in order to get the boundedness of ess sup $u$. Similar argument gives that ess inf $u$ is bounded.

Since (14) holds, by standard elliptic regularity theory (see e.g. [LU]) the eigenfunction is $\mathrm{C}^{1, \alpha}(\Omega)$.

\section{THE SECOND DIRICHLET EIGENVALUE OF $-\mathcal{Q}_{p}$}

If $\Omega$ is a bounded domain, Proposition 3.5 assures that the first eigenvalue $\lambda_{1}(p, \Omega)$ of (I) is isolated. This suggests the following definition. 
Definition 3. Let $\Omega$ be a bounded open set of $\mathbb{R}^{n}$. Then the second eigenvalue of $-Q_{p}$ is

$$
\lambda_{2}(p, \Omega):= \begin{cases}\min \left\{\lambda>\lambda_{1}(p, \Omega): \lambda \text { is an eigenvalue }\right\} & \text { if } \lambda_{1}(p, \Omega) \text { is simple } \\ \lambda_{1}(p, \Omega) & \text { otherwise. }\end{cases}
$$

Remark 4.1. If $\Omega$ is connected, by theorems 3.1 and 3.2 we deduce the following characterization of the second eigenvalue:

$\lambda_{2}(p, \Omega)=\min \{\lambda: \lambda$ admits a sign-changing eigenfunction $\}$.

We point out that in $[\mathrm{F}]$ it is proved that in a bounded open set it holds

$$
\lambda_{2}(p, \Omega)=\tilde{\lambda}_{2}(p, \Omega)=\inf _{\gamma \in \Gamma_{\Omega}\left(u_{1},-u_{1}\right)} \max _{\mathfrak{u} \in \gamma([0,1])} \int_{\Omega} F^{p}(\nabla u(x)) d x
$$

where $\tilde{\lambda}_{2}(p, \Omega)$ is given in Proposition 3.6 , and

$$
\Gamma_{\Omega}(u, v)=\{\gamma:[0,1] \rightarrow M: \gamma \text { is continuous and } \gamma(0)=u, \gamma(1)=v\},
$$

with $M$ as in (12). As immediate consequence of (18) we get

Proposition 4.2. If $\Omega_{1} \subseteq \Omega_{2} \subseteq \Omega$, then $\lambda_{2}\left(p, \Omega_{1}\right) \geqslant \lambda_{2}\left(p, \Omega_{2}\right)$.

By adapting the method contained in [CDG1, CDG2], it is possible to prove the following result.

Proposition 4.3. Let $\Omega$ be a bounded domain in $\mathbb{R}^{n}$. The eigenfunctions associated to $\lambda_{2}(p, \Omega)$ admit exactly two nodal domains.

Proof. We will proceed as in the proof of [CDG2, Th. 2.1]. In such a case, $\lambda_{2}(p, \Omega)$ is characterized as in (17). Then any eigenfunction $u_{2}$ has to change sign, and it admits at least two nodal domains $\Omega_{1} \subset \Omega^{+}$and $\Omega_{2} \subset \Omega^{-}$. Let us assume, by contradiction, the existence of a third nodal domain $\Omega_{3}$ and let us suppose, without loss of generality, that $\Omega_{3} \subset \Omega^{+}$.

Claim. There exists a connected open set $\widetilde{\Omega}_{2}$, with $\Omega_{2} \subset \widetilde{\Omega}_{2} \subset \Omega$ such that $\widetilde{\Omega}_{2} \cap \Omega_{1}=\emptyset$ or $\widetilde{\Omega}_{2} \cap \Omega_{3}=\emptyset$.

The proof of the claim follows line by line as in [CDG2, Th. 2.1]. One of the main tool is the Hopf maximum principle, that for the operator $-Q_{p}$ is proved for example in [CT, Th. 2.1].

Now, without loss of generality, we assume that $\widetilde{\Omega}_{2}$ is disjoint of $\Omega_{1}$ and from this fact a contradiction is derived.

By the fact that $u_{2}$ does not change sign on the nodal domains and by Proposition 4.2, we have that $\lambda_{1}\left(p, \Omega_{1}\right)=\lambda_{2}(p, \Omega)$ and that $\lambda_{1}\left(p, \widetilde{\Omega}_{2}\right)<\lambda_{1}\left(p, \Omega_{2}\right)=\lambda_{2}(p, \Omega)$. Now, we may construct the disjoint sets $\widetilde{\widetilde{\Omega}}_{2}$ and $\widetilde{\Omega}_{1}$ such that $\Omega_{2} \subset \widetilde{\widetilde{\Omega}}_{2} \subset \widetilde{\Omega}_{2}$ and $\Omega_{1} \subset \widetilde{\Omega}_{1}$, in order to have

$$
\lambda_{1}\left(p, \widetilde{\Omega}_{1}\right)<\lambda_{2}(p, \Omega), \quad \lambda_{1}\left(p, \widetilde{\widetilde{\Omega}}_{2}\right)<\lambda_{2}(p, \Omega) .
$$

Now let $v_{1}$ and $v_{2}$ be the extension by zero outside $\widetilde{\Omega}_{1}$ and $\widetilde{\widetilde{\Omega}}_{2}$, respectively, of the positive normalized eigenfunctions associated to $\lambda_{1}\left(p, \widetilde{\Omega}_{1}\right)$ and $\lambda\left(p, \widetilde{\Omega}_{2}\right)$. Hence we easily verify that the function $v=v_{1}-v_{2}$ belongs to $W_{0}^{1, p}(\Omega)$, it changes sign and satisfies

$$
\frac{\int_{\Omega} F^{p}\left(\nabla v_{+}\right) d x}{\int_{\Omega} v_{+}^{p} d x}<\lambda_{2}(p, \Omega), \quad \frac{\int_{\Omega} F^{p}\left(\nabla v_{-}\right) d x}{\int_{\Omega} v_{-}^{p} d x}<\lambda_{2}(p, \Omega) .
$$


The final aim is to construct a path $\gamma([0,1])$ such

$$
\max _{u \in \gamma([0,1])} \int_{\Omega} F^{p}(\nabla u(x)) d x<\lambda_{2}(p, \Omega),
$$

obtaining a contradiction from (18). The construction of this path follows adapting the method contained in [CDG1, CDG2].

Remark 4.4. In order to better understand the behavior of $\lambda_{1}(p, \Omega)$ and $\lambda_{2}(p, \Omega)$ on disconnected sets, an meaningful model is given when

$$
\Omega=\mathcal{W}_{\mathrm{r}_{1}} \cup \mathcal{W}_{\mathrm{r}_{2}} \text {, with } \mathrm{r}_{1}, \mathrm{r}_{2}>0 \text { and } \mathcal{W}_{\mathrm{r}_{1}} \cap \mathcal{W}_{\mathrm{r}_{2}}=\emptyset
$$

We distinguish two cases.

CASE $r_{1}<r_{2}$. We have

$$
\lambda_{1}(\mathrm{p}, \Omega)=\lambda_{1}\left(\mathrm{p}, \mathcal{W}_{\mathrm{r}_{2}}\right) .
$$

Hence $\lambda_{1}(p, \Omega)$ is simple, and any eigenfunction is identically zero on $\mathcal{W}_{1}$ and has constant sign in $\mathcal{W}_{2}$. Moreover,

$$
\lambda_{2}(p, \Omega)=\min \left\{\lambda_{1}\left(p, \mathcal{W}_{\mathrm{r}_{1}}\right), \lambda_{2}\left(p, \mathcal{W}_{\mathrm{r}_{2}}\right)\right\}
$$

Hence, if $r_{1}$ is not too small, then the second eigenvalue is $\lambda_{1}\left(\mathcal{W}_{r_{1}}\right)$, and the second eigenfunctions of $\Omega$ coincide with the first eigenfunctions of $\mathcal{W}_{\mathrm{r}_{1}}$, that do not change sign in $\mathcal{W}_{\mathrm{r}_{1}}$, and vanish on $\mathcal{W}_{\mathrm{r}_{2}}$.

CASE $r_{1}=r_{2}$. We have

$$
\lambda_{1}(p, \Omega)=\lambda_{1}\left(p, \mathcal{W}_{r_{i}}\right), \quad i=1,2 .
$$

The first eigenvalue $\lambda_{1}(p, \Omega)$ is not simple: choosing, for example, the function $U=u_{1} \chi \mathcal{W}_{r_{1}}-$ $u_{2} \chi \mathcal{W}_{r_{2}}$, where $u_{i}, i=1,2$, is the first normalized eigenfunction of $\lambda_{1}\left(p, \mathcal{W}_{r_{i}}\right)$, and $v=$ $u_{1} X \mathcal{W}_{r_{1}}$, then $U$ and $V$ are two nonproportional eigenfunctions relative to $\lambda_{1}(p, \Omega)$. Hence, in this case, by definition,

$$
\lambda_{2}(p, \Omega)=\lambda_{1}(p, \Omega)=\lambda_{1}\left(p, \mathcal{W}_{r_{i}}\right) .
$$

In order to prove the Hong-Krahn-Szego inequality, we need the following key lemma.

Proposition 4.5. Let $\Omega$ be an open bounded set of $\mathbb{R}^{\mathrm{n}}$. Then there exists two disjoint domains $\Omega_{1}, \Omega_{2}$ of $\Omega$ such that

$$
\lambda_{2}(p, \Omega)=\max \left\{\lambda_{1}\left(p, \Omega_{1}\right), \lambda_{1}\left(p, \Omega_{2}\right)\right\} .
$$

Proof. Let $u_{2} \in W_{0}^{1, p}(\Omega)$ be a second normalized eigenfunction. First of all, suppose that $u_{2}$ changes sign in $\Omega$. Then, consider two nodal domains $\Omega_{1} \subseteq \Omega_{+}$and $\Omega_{2} \subseteq \Omega_{-}$. By definition, $\Omega_{1}$ and $\Omega_{2}$ are connected sets. The restriction of $u_{2}$ to $\Omega_{1}$ is, by Theorem 3.2, a first eigenfunction for $\Omega_{1}$ and hence $\lambda_{2}(p, \Omega)=\lambda_{1}\left(p, \Omega_{1}\right)$. Analogously for $\Omega_{2}$, hence

$$
\lambda_{2}(p, \Omega)=\lambda_{1}\left(p, \Omega_{1}\right)=\lambda_{1}\left(p, \Omega_{2}\right),
$$

and the proof of the proposition is completed, in the case $u_{2}$ changes sign. 
In the case that $u_{2}$ has constant sign in $\Omega$, for example $u_{2} \geqslant 0$, then by Theorem $3.2 \Omega$ must be disconnected. If $\lambda_{1}(p, \Omega)$ is simple, by definition $\lambda_{2}(p, \Omega)>\lambda_{1}(p, \Omega)$. Otherwise, $\lambda_{1}(p, \Omega)=\lambda_{2}(p, \Omega)$. Hence in both cases, we can consider a first nonnegative normalized eigenfunction $u_{1}$ not proportional to $u_{2}$.

Observe that in any connected component of $\Omega$, by the Harnack inequality, $u_{i}, i=1,2$, must be positive or identically zero. Hence we can choose two disjoint connected open sets $\Omega_{1}$ and $\Omega_{2}$, contained respectively in $\left\{x \in \Omega: u_{1}(x)>0\right\}$ and $\left\{x \in \Omega: u_{2}(x)>0\right\}$. Then, $u_{1}$ and $u_{2}$ are first Dirichlet eigenfunctions in $\Omega_{1}$ and $\Omega_{2}$, respectively, and

$$
\lambda_{1}(p, \Omega)=\lambda_{1}\left(p, \Omega_{1}\right) \leqslant \lambda_{2}(p, \Omega), \quad \lambda_{2}(p, \Omega)=\lambda_{1}\left(p, \Omega_{2}\right),
$$

and the proof is completed.

Now we are in position to prove the Hong-Krahn-Szego inequality for $\lambda_{2}(p, \Omega)$.

Theorem 4.6. Let $\Omega$ be a bounded open set of $\mathbb{R}^{\mathrm{n}}$. Then

$$
\lambda_{2}(p, \Omega) \geqslant \lambda_{2}(p, \widetilde{\mathcal{W}}),
$$

where $\widetilde{\mathcal{W}}$ is the union of two disjoint Wulff shapes, each one of measure $\frac{|\Omega|}{2}$. Moreover equality sign in (19) occurs if $\Omega$ is the disjoint union of two Wulff shapes of the same measure.

Proof. Let $\Omega_{1}$ and $\Omega_{2}$ given by Proposition 4.5. By the Faber-Krahn inequality we have

$$
\lambda_{2}(p, \Omega)=\max \left\{\lambda_{1}\left(p, \Omega_{1}\right), \lambda_{1}\left(p, \Omega_{2}\right)\right\} \geqslant \max \left\{\lambda_{1}\left(p, \mathcal{W}_{r_{1}}\right), \lambda_{1}\left(p, \mathcal{W}_{r_{2}}\right)\right\}
$$

with $\left|\mathcal{W}_{r_{\mathfrak{i}}}\right|=\left|\Omega_{\mathfrak{i}}\right|$. By the rescaling property of $\lambda_{1}(\mathrm{p}, \cdot)$, and observing that, being $\Omega_{1}$ and $\Omega_{2}$ disjoint subsets of $\Omega,\left|\Omega_{1}\right|+\left|\Omega_{2}\right| \leqslant|\Omega|$, we have that

$$
\begin{aligned}
\max \left\{\lambda_{1}\left(p, \mathcal{W}_{r_{1}}\right), \lambda_{1}\left(p, \mathcal{W}_{r_{2}}\right)\right\}=\lambda_{1}(p, \mathcal{W}) \kappa_{n}^{\frac{p}{n}} \max \left\{\left|\Omega_{1}\right|^{-\frac{p}{n}},\left|\Omega_{2}\right|^{-\frac{p}{n}}\right\} \geqslant \\
\geqslant \lambda_{1}(p, \mathcal{W}) \kappa_{n}^{\frac{p}{n}}\left(\frac{|\Omega|}{2}\right)^{-\frac{p}{n}}=\lambda_{1}(p, \widetilde{\mathcal{W}}) .
\end{aligned}
$$

\section{THE LIMIT CASE $p \rightarrow \infty$}

In this section we derive some information on $\lambda_{2}(p, \Omega)$ as $p$ goes to infinity. First of all we recall some known result about the limit of the first eigenvalue. Let us consider a bounded open set $\Omega$.

The anisotropic distance of $x \in \bar{\Omega}$ to the boundary of $\Omega$ is the function

$$
\mathrm{d}_{\mathrm{F}}(x)=\inf _{y \in \partial \Omega} \mathrm{F}^{\mathrm{o}}(x-y), \quad x \in \bar{\Omega} .
$$

We stress that when $F=|\cdot|$ then $d_{F}=d_{\mathcal{E}}$, the Euclidean distance function from the boundary.

It is not difficult to prove that $d_{F}$ is a uniform Lipschitz function in $\bar{\Omega}$ and

$$
\mathrm{F}\left(\nabla \mathrm{d}_{\mathrm{F}}(\mathrm{x})\right)=1 \text { a.e. in } \Omega \text {. }
$$

Obviously, $d_{F} \in W_{0}^{1, \infty}(\Omega)$. Let us consider the quantity

$$
\rho_{\mathrm{F}}=\max \left\{\mathrm{d}_{\mathrm{F}}(x), x \in \bar{\Omega}\right\} .
$$

If $\Omega$ is connected, $\rho_{\mathrm{F}}$ is called the anisotropic inradius of $\Omega$. If not, $\rho_{\mathrm{F}}$ is the maximum of the inradii of the connected components of $\Omega$.

For further properties of the anisotropic distance function we refer the reader to [CM]. 
Remark 5.1. It is easy to prove (see also [JLM, BKJ]) that the distance function satisfies

$$
\frac{1}{\rho_{\mathrm{F}}(\Omega)}=\frac{1}{\left\|\mathrm{~d}_{\mathrm{F}}\right\|_{\mathrm{L}^{\infty}(\Omega)}}=\min _{\varphi \in W_{0}^{1, \infty}(\Omega) \backslash\{0\}} \frac{\|\mathrm{F}(\nabla \varphi)\|_{\mathrm{L}^{\infty}(\Omega)}}{\|\varphi\|_{\mathrm{L}^{\infty}(\Omega)}} .
$$

Indeed it is sufficient to observe that if $\varphi \in C_{0}^{1}(\Omega) \cap C(\bar{\Omega})$, then $\varphi \in C_{0}^{1}\left(\Omega_{i}\right) \cap C\left(\bar{\Omega}_{i}\right)$, for any connected component $\Omega_{i}$ of $\Omega$. Then for a.e. $x \in \Omega_{i}$, for $y \in \partial \Omega_{i}$ which achieves $\mathrm{F}^{\mathrm{o}}(x-y)=\mathrm{d}_{\mathrm{F}}(x)$, it holds

$$
\begin{aligned}
|\varphi(x)|=|\varphi(x)-\varphi(y)|=|\langle\nabla \varphi(\xi), x-y\rangle| & \leqslant \\
& \leqslant F(\nabla \varphi(\xi)) F^{o}(x-y) \leqslant\|F(\nabla \varphi)\|_{L^{\infty}(\Omega)} d_{F}(x) .
\end{aligned}
$$

Passing to the supremum and by density we get (20).

The following result holds (see [BKJ, JLM]).

Theorem 5.2. Let $\Omega$ be a bounded domain in $\mathbb{R}^{n}$, and let $\lambda_{1}(p, \Omega)$ be the first eigenvalue of $(7)$. Then

$$
\lim _{p \rightarrow \infty} \lambda_{1}(p, \Omega)^{\frac{1}{p}}=\frac{1}{\rho_{\mathrm{F}}(\Omega)} .
$$

Now let us define

$$
\Lambda_{1}(\infty, \Omega)=\frac{1}{\rho_{\mathrm{F}}(\Omega)} .
$$

The value $\Lambda_{1}(\infty, \Omega)$ is related to the so-called anisotropic infinity Laplacian operator defined in $[\mathrm{BKJ}]$, that is

$$
2_{\infty} \mathfrak{u}=\left\langle\nabla^{2} \mathrm{uJ}(\nabla \mathrm{u}), \mathrm{J}(\nabla \mathrm{u})\right\rangle,
$$

where $\mathrm{J}(\xi)=\frac{1}{2} \nabla_{\xi}\left[\mathrm{F}^{2}\right](\xi)$. Note that we mean, by continuous extension, $\mathrm{J}(0)=0$. This is possible being $\mathrm{F}$ 1-homogeneous and $\mathrm{F}(0)=0$.

Indeed, in $[\mathrm{BKJ}]$ the following result is proved.

Theorem 5.3. Let $\Omega$ be a bounded domain in $\mathbb{R}^{n}$. Then, there exists a positive solution $\mathrm{u}_{\infty} \in$ $W_{0}^{1, \infty}(\Omega) \cap \mathrm{C}(\bar{\Omega})$ which satisfies, in the viscosity sense, the following problem:

$$
\begin{cases}\min \left\{F(\nabla u)-\Lambda u,-Q_{\infty} u\right\}=0 & \text { in } \Omega, \\ u=0 & \text { on } \partial \Omega .\end{cases}
$$

with $\Lambda=\Lambda_{1}(\infty, \Omega)$. Moreover, any positive solution $v \in W_{0}^{1, \infty}(\Omega)$ to $(21)$ with $\Lambda=\Lambda_{1}(\infty, \Omega)$ satisfies

$$
\frac{\|F(\nabla v)\|_{L^{\infty}(\Omega)}}{\|v\|_{L^{\infty}(\Omega)}}=\min _{\varphi \in W_{0}^{1, \infty}(\Omega) \backslash\{0\}} \frac{\|F(\nabla \varphi)\|_{L^{\infty}(\Omega)}}{\|\varphi\|_{L^{\infty}(\Omega)}}=\Lambda_{1}(\infty, \Omega)=\frac{1}{\rho_{F}(\Omega)} .
$$

Finally, if problem (21) admits a positive viscosity solution in $\Omega$, then $\Lambda=\Lambda_{1}(\infty, \Omega)$.

Proposition 5.4. Theorem 5.2 holds also when $\Omega$ is a bounded open set of $\mathbb{R}^{n}$.

Proof. Suppose that $\Omega$ is not connected, and consider a connected component $\Omega_{0}$ of $\Omega$ with anisotropic inradius $\rho_{F}(\Omega)$. By the monotonicity property of $\lambda_{1}(p, \Omega)$ given in Proposition $3 \cdot 3$, we have

$$
\lambda_{1}(p, \Omega) \leqslant \lambda_{1}\left(p, \Omega_{0}\right) .
$$


Then up to a subsequence, passing to the limit as $p \rightarrow+\infty$ and using Theorem 5.2 we have

$$
\tilde{\Lambda}=\lim _{p_{j} \rightarrow \infty} \lambda_{1}\left(p_{j}, \Omega\right)^{\frac{1}{p_{j}}} \leqslant \frac{1}{\rho_{F}(\Omega)} .
$$

In order to prove that $\tilde{\Lambda}=\rho_{\mathrm{F}}(\Omega)^{-1}$, let $u_{p_{j}}$ the first nonnegative normalized eigenfunction associated to $\lambda_{1}\left(p_{j}, \Omega\right)$. Reasoning as in [BKJ], the sequence $u_{p_{j}}$ converges to a function $u_{\infty}$ in $C^{0}(\Omega)$ which is a viscosity solution of $(21)$ associated to $\tilde{\Lambda}$. Then by the maximum principle contained in [BB, Lemma 3.2], in each connected component of $\Omega, u_{\infty}$ is either positive or identically zero. Denoting by $\tilde{\Omega}$ a connected component of $\left\{u_{\infty}>0\right\}$, by the uniform convergence, for $p_{j}$ large, also $u_{p_{j}}$ is positive in $\tilde{\Omega}$. Then by Theorem 3.2 we have

$$
\lambda_{1}\left(p_{j}, \tilde{\Omega}\right)=\lambda_{1}\left(p_{j}, \Omega\right), \quad \text { and then } \frac{1}{\rho_{F}(\tilde{\Omega})}=\tilde{\Lambda}
$$

By (22) and by definition of $\rho_{\mathrm{F}}, \tilde{\Lambda} \leqslant \rho_{\mathrm{F}}(\Omega)^{-1} \leqslant \rho_{\mathrm{F}}(\tilde{\Omega})^{-1}=\tilde{\Lambda}$; then necessarily $\tilde{\Lambda}=\rho_{\mathrm{F}}(\Omega)^{-1}$.

In order to define the eigenvalue problem for $Q_{\infty}$, let us consider the following operator

$$
\mathcal{A}_{\wedge}(s, \xi, X)= \begin{cases}\min \{\mathrm{F}(\xi)-\wedge s,-\langle\mathrm{XJ}(\xi), J(\xi)\rangle\} & \text { if } s>0, \\ -\langle\mathrm{XJ}(\xi), J(\xi)\rangle & \text { if } s=0, \\ \max \{-\mathrm{F}(\xi)-\wedge s,-\langle\mathrm{XJ}(\xi), J(\xi)\rangle\} & \text { if } s<0,\end{cases}
$$

with $(s, \xi, X) \in \mathbb{R} \times \mathbb{R}^{n} \times S^{n \times n}$, where $S^{n \times n}$ denotes the space of real, symmetric matrices of order $n$. Clearly $\mathcal{A}_{\wedge}$ is not continuous in $s=0$.

For completeness we recall the definition of viscosity solution for the operator $\mathcal{A}_{\Lambda}$.

Definition 4. Let $\Omega \subset \mathbb{R}^{\mathrm{n}}$ a bounded open set. A function $\mathrm{u} \in \mathrm{C}(\Omega)$ is a viscosity subsolution (resp. supersolution) of $\mathcal{A}_{\wedge}(\mathrm{x}, \mathrm{u}, \nabla \mathrm{u})=0$ if

$$
\mathcal{A}_{\wedge}\left(\phi(x), \nabla \phi(x), \nabla^{2} \phi(x)\right) \leqslant 0 \quad\left(\operatorname{resp} . \mathcal{A}_{\wedge}\left(\phi(x), \nabla \phi(x), \nabla^{2} \phi(x)\right) \geqslant 0\right),
$$

for every $\phi \in \mathrm{C}^{2}(\Omega)$ such that $\mathrm{u}-\phi$ has a local maximum (resp. minimum) zero at $\mathrm{x}$. A function $\mathrm{u} \in \mathrm{C}(\Omega)$ is a viscosity solution of $\mathcal{A}_{\Lambda}=0$ if it is both a viscosity subsolution and a viscosity supersolution and in this case the number $\Lambda$ is called an eigenvalue for $Q_{\infty}$.

Definition 5. We say that $\mathrm{u} \in \mathrm{C}(\bar{\Omega}),\left.\mathrm{u}\right|_{\partial \Omega}=0, \mathrm{u} \not \equiv 0$ is an eigenfunction for the anisotropic $\infty$-Laplacian if there exists $\Lambda \in \mathbb{R}$ such that

$$
\mathcal{A}_{\wedge}\left(\mathrm{u}, \nabla \mathrm{u}, \nabla^{2} \mathrm{u}\right)=0 \quad \text { in } \Omega
$$

in the viscosity sense. Such value $\Lambda$ will be called an eigenvalue for the anisotropic $\infty$-Laplacian.

In order to define the second eigenvalue for $Q_{\infty}$ we introduce the following number:

$$
\rho_{2, \mathrm{~F}}(\Omega)=\sup \left\{\rho>0 \text { : there are two disjoint Wulff shapes } \mathcal{W}_{1}, \mathcal{W}_{2} \subset \Omega \text { of radius } \rho\right\},
$$

and let us define

$$
\Lambda_{2}(\infty, \Omega)=\frac{1}{\rho_{2, \mathrm{~F}}(\Omega)} .
$$

\section{Clearly}

$$
\Lambda_{1}(\infty, \Omega) \leqslant \Lambda_{2}(\infty, \Omega) .
$$


Remark 5.5. It is easy to construct open sets $\Omega$ such that $\Lambda_{1}(\infty, \Omega)=\Lambda_{2}(\infty, \Omega)$. For example, this holds when $\Omega$ coincides with the union of two disjoint Wulff shapes with same measure, or their convex envelope.

Remark 5.6. A simple example of $\rho_{2, \mathrm{~F}}(\Omega)$ is given when $\Omega$ is the union of two disjoint Wulff sets, $\Omega=\mathcal{W}_{r_{1}} \cup W_{r_{2}}$, with $r_{2} \leqslant r_{1}$. In this case, $\Lambda_{1}(\infty, \Omega)=\frac{1}{r_{1}}$ and, if $r_{2}$ is not too small, then $\Lambda_{2}(\infty, \Omega)=\frac{1}{r_{2}}$.

Theorem 5.7. Let $\Omega \subset \mathbb{R}^{n}$ be a bounded open set and let $\lambda_{2}(p, \Omega)$ be the second Dirichlet eigenvalue of $-Q_{p}$ in $\Omega$. Then

$$
\lim _{p \rightarrow \infty} \lambda_{2}(p, \Omega)^{\frac{1}{p}}=\Lambda_{2}(\infty, \Omega)=\frac{1}{\rho_{2, F}(\Omega)} .
$$

Moreover $\Lambda_{2}(\infty, \Omega)$ is an eigenvalue of $Q_{\infty}$, that is $\Lambda_{2}(\infty, \Omega)$ is an eigenvalue for the anisotropic infinity Laplacian in the sense of Definition 5.

Proof. First we observe that $\lambda_{2}(p, \Omega)^{\frac{1}{p}}$ is bounded from above with respect to $p$. More precisely we have

$$
\Lambda_{1}(\infty, \Omega) \leqslant \limsup _{p \rightarrow \infty} \lambda_{2}(p, \Omega)^{\frac{1}{p}} \leqslant \Lambda_{2}(\infty, \Omega) .
$$

Indeed if we consider two disjoint Wulff shapes $\mathcal{W}_{1}$ and $\mathcal{W}_{2}$ of radius $\rho_{2, \mathrm{~F}}(\Omega)$, clearly $\mathcal{W}_{1} \cup$ $\mathcal{W}_{2} \subset \Omega$ and then by monotonicity property (Proposition 4.2) of $\lambda_{2}(p, \Omega)$ we have

$$
\lambda_{1}(p, \Omega)^{\frac{1}{p}} \leqslant \lambda_{2}(p, \Omega)^{\frac{1}{p}} \leqslant \lambda_{2}\left(p, \mathcal{W}_{1} \cup \mathcal{W}_{2}\right)^{\frac{1}{p}}=\lambda_{1}\left(p, \mathcal{W}_{1}\right)^{\frac{1}{p}},
$$

where last equality follows from Remark 4.4. Then passing to the limit as $p \rightarrow \infty$ in the right hand side, by Theorem 5.2 we have (24). Hence there exists a sequence $p_{j}$ such that $p_{j} \rightarrow+\infty$ as $j \rightarrow \infty$, and

$$
\frac{1}{\rho_{\mathrm{F}}(\Omega)}=\Lambda_{1}(\infty, \Omega) \leqslant \lim _{j \rightarrow \infty} \lambda_{2}\left(p_{j}, \Omega\right)^{\frac{1}{p_{j}}}=\bar{\Lambda} \leqslant \Lambda_{2}(\infty, \Omega)=\frac{1}{\rho_{2, \mathrm{~F}}(\Omega)} .
$$

In order to conclude the proof we have to show that $\bar{\Lambda}$ is an eigenvalue for $Q_{\infty}$ and that $\bar{\Lambda}=\Lambda_{2}(\infty, \Omega)$.

Let us consider $u_{j} \in W_{0}^{1, p}(\Omega)$ eigenfunction of $\lambda_{2}\left(p_{j}, \Omega\right)$ such that $\left\|u_{j}\right\|_{L^{p_{j}}(\Omega)}=1$. Then by standard arguments $u_{j}$, converges, up to a subsequence of $p_{j}$, uniformly to a function $u \in W_{0}^{1, \infty}(\Omega) \cap \mathrm{C}(\bar{\Omega})$. The function $u$ is a viscosity solution of (23) with $\Lambda=\bar{\Lambda}$. Indeed, let $x_{0} \in \Omega$. If $u\left(x_{0}\right)>0$, being $u$ continuous, it is positive in a sufficiently small ball centered at $x_{0}$. Then it is possible to proceed exactly as in $[\mathrm{BKJ}]$ in order to obtain that, in the viscosity sense,

$$
\min \left\{\mathrm{F}\left(\nabla \mathfrak{u}\left(\mathrm{x}_{0}\right)\right)-\bar{\Lambda} \mathfrak{u}\left(\mathrm{x}_{0}\right),-Q_{\infty} \mathfrak{u}\left(\mathrm{x}_{0}\right)\right\}=0 .
$$

Similarly, if $u\left(x_{0}\right)<0$ then

$$
\max \left\{-\mathrm{F}\left(\nabla \mathrm{u}\left(\mathrm{x}_{0}\right)\right)-\bar{\Lambda} \mathrm{u}\left(\mathrm{x}_{0}\right),-\mathcal{Q}_{\infty} \mathrm{u}\left(\mathrm{x}_{0}\right)\right\}=0 .
$$

It remains to consider the case $u\left(x_{0}\right)=0$. We will show that $u$ is a subsolution of (23).

Let $\varphi$ a $\mathrm{C}^{2}(\Omega)$ function such that $u-\varphi$ has a strict maximum point at $x_{0}$. By the definition of $\mathcal{A}_{\bar{\Lambda}}$, we have to show that $-Q_{\infty} \varphi\left(x_{0}\right) \leqslant 0$. 
For any $j$, let $x_{j}$ be a maximum point of $u_{j}-\varphi$, so that $x_{j} \rightarrow x_{0}$ as $j \rightarrow \infty$. Such sequence exists by the uniform convergence of $u_{j}$. By [BKJ, Lemma 2.3] $u_{j}$ verifies in the viscosity sense $-Q_{p} u_{j}=\lambda_{2}\left(p_{j}, \Omega\right)\left|u_{j}\right|^{p_{j}-2} u_{j}$. Then

$$
\begin{aligned}
& -Q_{p} \varphi_{j}\left(x_{j}\right)= \\
& =-\left(p_{j}-2\right) F^{p_{j}-4}\left(\nabla \varphi\left(x_{j}\right)\right)\left\langle\nabla^{2} \varphi\left(x_{j}\right) J\left(\nabla \varphi\left(x_{j}\right)\right), J\left(\nabla \varphi\left(x_{j}\right)\right)\right\rangle+ \\
& \quad-F^{p_{j}-2}\left(\nabla \varphi\left(x_{j}\right)\right) \nabla^{2} \varphi\left(x_{j}\right) \otimes \nabla_{\xi} J\left(\nabla \varphi\left(x_{j}\right)\right)= \\
& =-\left(p_{j}-2\right) F^{p_{j}-4}\left(\nabla \varphi\left(x_{j}\right)\right) Q_{\infty} \varphi\left(x_{j}\right)-F^{p_{j}-2}\left(\nabla \varphi\left(x_{j}\right)\right) Q_{2} \varphi\left(x_{j}\right) \leqslant \\
& \leqslant \lambda_{2}\left(p_{j}, \Omega\right)\left|u_{j}\left(x_{j}\right)\right|^{p_{j}-2} u_{j}\left(x_{j}\right) ;
\end{aligned}
$$

here $A \otimes B:=\sum_{i, k} A_{i k} B_{i k}$, for two $n \times n$ matrices $A$, B. If $\nabla \varphi\left(x_{0}\right) \neq 0$, then dividing the above inequality by $\left(\mathrm{p}_{j}-2\right) \mathrm{Fp}^{p_{j}-4}(\nabla \varphi)$ we have

$$
-Q_{\infty} \varphi\left(x_{j}\right) \leqslant \frac{F^{2}\left(\nabla \varphi\left(x_{j}\right)\right) Q_{2} \varphi\left(x_{j}\right)}{p_{j}-2}+\left(\frac{\lambda_{2}\left(p_{j}, \Omega\right)^{\frac{1}{p_{j}-4}}\left|u_{j}\left(x_{j}\right)\right|}{F\left(\nabla \varphi\left(x_{j}\right)\right)}\right)^{p_{j}-4} \frac{u_{j}\left(x_{j}\right)^{3}}{p_{j}-2}=: \ell_{j} .
$$

Passing to the limit as $j \rightarrow \infty$, recalling that $\varphi \in C^{2}(\Omega), F \in C^{2}\left(\mathbb{R}^{\mathfrak{n}} \backslash\{0\}\right), \lambda_{2}\left(p_{j}, \Omega\right)^{\frac{1}{p_{j}}} \rightarrow \bar{\Lambda}$, $\nabla \varphi\left(x_{0}\right) \neq 0$ and $u_{\mathfrak{j}}\left(x_{\mathfrak{j}}\right) \rightarrow 0$ we get

$$
-Q_{\infty} \varphi\left(x_{0}\right) \leqslant 0
$$

Finally, we note that if $\nabla \varphi\left(x_{0}\right)=0$, the above inequality is trivially true. Hence, we can conclude that $u$ is a viscosity subsolution.

The proof that $u$ is also a viscosity supersolution can be done by repeating the same argument than before, considering $-\mathfrak{u}$.

Last step of the proof of the Theorem consists in showing that $\bar{\Lambda}=\Lambda_{2}(\infty, \Omega)$. We distinguish two cases.

Case 1: The function $u$ changes sign in $\Omega$.

Let us consider the following sets

$$
\Omega^{+}=\{x \in \Omega: u(x)>0\} \quad \Omega^{-}=\{x \in \Omega: u(x)<0\} .
$$

Being $u \in C^{0}(\Omega)$ then $\Omega^{+}, \Omega^{-}$are two disjoint open sets of $\mathbb{R}^{n}$ and $\left|\Omega^{+}\right|>0$ and $\left|\Omega^{-}\right|>0$.

By Theorem 5.3 we have

$$
\bar{\Lambda}=\Lambda_{1}\left(\infty, \Omega^{+}\right) \quad \text { and } \quad \bar{\Lambda}=\Lambda_{1}\left(\infty, \Omega^{-}\right) .
$$

Then by definition of $\rho_{2, F}$ we get

$$
\rho_{\mathrm{F}}\left(\Omega^{+}\right)=\rho_{\mathrm{F}}\left(\Omega^{-}\right)=\frac{1}{\bar{\Lambda}} \leqslant \rho_{2, \mathrm{~F}}(\Omega),
$$

that implies, by (25) that

$$
\bar{\Lambda}=\Lambda_{2}(\infty, \Omega) \text {. }
$$

Case 2: The function $u$ does not change sign in $\Omega$.

We first observe that in this case $\Omega$ cannot be connected. Indeed since $u_{j}$ converges to $u$ in $\mathrm{C}^{0}(\bar{\Omega})$, for sufficiently large $p$ we have that there exist second eigenfunctions relative to $\lambda_{2}(p, \Omega)$ with constant sign in $\Omega$ and this cannot happen if $\Omega$ is connected. 
Then in this case, we have to replace the sequence $u_{j}$ (and then the function $u$ ) in order to find two disjoint connected open subsets $\Omega_{1}, \Omega_{2}$ of $\Omega$, such that

$$
\Lambda_{1}(\infty, \Omega)=\Lambda_{1}\left(\infty, \Omega_{1}\right)
$$

and

$$
\bar{\Lambda}=\Lambda_{1}\left(\infty, \Omega_{2}\right) .
$$

Once we prove that such subsets exist, by $(25)$ and the definition of $\rho_{2, \mathrm{~F}}$ we obtain

$$
\rho_{\mathrm{F}}\left(\Omega_{2}\right)=\frac{1}{\bar{\Lambda}} \leqslant \rho_{2, \mathrm{~F}}(\Omega) \leqslant \rho_{\mathrm{F}}(\Omega)=\rho_{\mathrm{F}}\left(\Omega_{1}\right),
$$

that implies, again by (25),

$$
\bar{\Lambda}=\Lambda_{2}(\infty, \Omega) \text {. }
$$

In order to prove (26) and (27), we consider $u_{1, \infty}$, an eigenfunction associated to $\Lambda_{1}(\infty, \Omega)$, obtained as limit in $C^{0}(\Omega)$ of a sequence $u_{1, p}$ of first normalized eigenfunctions associated to $\lambda_{1}(p, \Omega)$, and consider a connected component of $\Omega$, say $\Omega_{1}$, where $u_{1, \infty}>0$ and such that $\Lambda_{1}(\infty, \Omega)=\Lambda_{1}\left(\infty, \Omega_{1}\right)$. The argument of the proof of Proposition 5.4 gives that such $u_{1, \infty}$ and $\Omega_{1}$ exist. Then, let $u_{2, p} \geqslant 0$ be a normalized eigenfunction associated to $\lambda_{2}(p, \Omega)$ such that for any $p$ sufficiently large, $\operatorname{spt}\left(u_{2, p}\right) \cap \Omega_{1}=\emptyset$.

The existence of such a sequence is guaranteed from this three observations:

- if $u_{2, p}$ changes sign for a divergent sequence of $p^{\prime} s$, then we come back to the case 1 ;

- by the maximum principle, in each connected component of $\Omega u_{2, p}$ is either positive or identically zero;

- the condition $\operatorname{spt}\left(\mathfrak{u}_{2, p}\right) \cap \Omega_{1}=\emptyset$ depends from the fact that $\mathfrak{u}_{2, p}$ can be chosen not proportional to $u_{1, p}$.

Hence, there exists $\Omega_{2}$ connected component of $\Omega$ disjoint from $\Omega_{1}$, such that $u_{2, p}$ converges to $u_{2, \infty}$ (up to a subsequence) in $C^{0}\left(\Omega_{2}\right)$, and where $u_{2, \infty}>0$. By Theorem 5.3, (27) holds.

Theorem 5.8. Given $\Omega$ bounded open set of $\mathbb{R}^{n}$, let $\Lambda>\Lambda_{1}(\infty, \Omega)$ be an eigenvalue for $Q_{\infty}$. Then $\Lambda \geqslant \Lambda_{2}(\infty, \Omega)$ and $\Lambda_{2}(\infty, \Omega)$ is the second eigenvalue of $Q_{\infty}$, in the sense that there are no eigenvalues of $Q_{\infty}$ between $\Lambda_{1}(\infty, \Omega)$ and $\Lambda_{2}(\infty, \Omega)$.

Proof. Let $u_{\wedge}$ be an eigenfunction corresponding to $\Lambda$. We distinguish two cases.

Case 1: The function $u_{\wedge}$ changes sign in $\Omega$.

Let us consider the following sets

$$
\Omega^{+}=\left\{x \in \Omega: u_{\Lambda}(x)>0\right\} \quad \Omega^{-}=\left\{x \in \Omega: u_{\Lambda}(x)<0\right\} .
$$

Being $u_{\Lambda} \in C^{0}(\Omega)$ then $\Omega^{+}, \Omega^{-}$are two disjoint open sets of $\mathbb{R}^{n}$ and $\left|\Omega^{+}\right|>0$ and $\left|\Omega^{-}\right|>0$.

By Theorem 5.3 we have

$$
\Lambda=\Lambda_{1}\left(\infty, \Omega^{+}\right) \text {and } \Lambda=\Lambda_{1}\left(\infty, \Omega^{-}\right) .
$$

Then by definition of $\rho_{2, F}$ we get

$$
\rho_{\mathrm{F}}\left(\Omega^{+}\right)=\rho_{\mathrm{F}}\left(\Omega^{-}\right)=\frac{1}{\Lambda} \leqslant \rho_{2, \mathrm{~F}}(\Omega),
$$


that implies, by (25) that

$$
\Lambda \geqslant \Lambda_{2}(\infty, \Omega) .
$$

Case 2: The function $u_{\wedge}$ does not change sign in $\Omega$.

By Theorem $5.3 \Omega$ cannot be connected being $\Lambda>\Lambda_{1}(\infty, \Omega)$.

In this case, again by By Theorem 5.3 we can find two disjoint connected open subsets $\Omega_{1}, \Omega_{2}$ of $\Omega$, such that

$$
\Lambda_{1}(\infty, \Omega)=\Lambda_{1}\left(\infty, \Omega_{1}\right)
$$

and

$$
\Lambda=\Lambda_{1}\left(\infty, \Omega_{2}\right) .
$$

Being $\Lambda>\Lambda_{1}(\infty, \Omega)$, we obtain

$$
\rho_{\mathrm{F}}\left(\Omega_{2}\right)=\frac{1}{\Lambda}<\rho_{\mathrm{F}}(\Omega)=\rho_{\mathrm{F}}\left(\Omega_{1}\right),
$$

that by the definition of $\rho_{2, F}$ implies,

$$
\Lambda \geqslant \Lambda_{2}(\infty, \Omega) .
$$

Remark 5.9. We observe that if $\Omega$ is a bounded open set and $\widetilde{\mathcal{W}}$ is the union of two disjoint Wulff sets with the same measure $|\Omega| / 2$, it holds that

$$
\rho_{2, \mathrm{~F}}(\Omega) \leqslant \rho_{2, \mathrm{~F}}(\widetilde{\mathcal{W}}),
$$

that is,

$$
\Lambda_{2}(\infty, \Omega) \geqslant \Lambda_{2}(\infty, \widetilde{\mathcal{W}}),
$$

that is the Hong-Krahn-Szego inequality for the second eigenvalue of $-Q_{\infty}$.

\section{ACNOWLEDGEMENTS}

This work has been partially supported by the FIRB 2013 project "Geometrical and qualitative aspects of PDE's" and by GNAMPA of INdAM.

\section{REFERENCES}

[AFLT] A.Alvino, V. Ferone, P.L. Lions, G. Trombetti. Convex symmetrization and applications. Ann. Inst. H. Poincaré Anal. non linéaire 14 (1997), 275-293. 6

[AFT] A.Alvino, V. Ferone, G. Trombetti. On the properties of some nonlinear eigenvalues. SIAM J. Math. Anal. 29 (1998), 437-451. 7

[BB] G. Barles, J. Busca. Existence and comparison results for fully nonlinear degenerate elliptic equations without zeroth order term. Comm. PDE 26 (2001): 2323-2337. 12

[BP] G. Bellettini, M. Paolini. Anisotropic motion by mean curvature in the context of Finsler geometry. Hokkaido Math. J., 25 (1996): 537-566. 4 
[BFK] M. Belloni, V. Ferone, B. Kawohl. Isoperimetric inequalities, Wulff shape and related questions for strongly nonlinear elliptic operators. Z. angew. Math. Phys. ZAMP 54.5 (2003): 771-783. 2, 5, 6

[BKJ] M. Belloni, B. Kawohl, P. Juutinen. The $p$-Laplace eigenvalue problem as $p \rightarrow \infty$ in a Finsler metric. J. Eur. Math. Soc. 8 (2006): 123-138. 2, 11, 12, 14

[BF2] L.Brasco, G. Franzina. On the Hong-Krahn-Szego inequality for the p-Laplace operator. Manuscripta Math., 141.3-4 (2013): 537-557. 2

[BGM] G. Buttazzo, S. Guarino Lo Bianco, M. Marini. Sharp estimates for the anisotropic torsional rigidity and the principal frequency. Preprint. 2

[CM] G.Crasta, A. Malusa, The distance function from the boundary in a Minkowski space, Trans. Amer. Math. Soc., 359 (2007), 5725-5759. II

[CDG1] M. Cuesta, D. De Figueiredo, J-P. Gossez. The beginning of the Fučik spectrum for the p-Laplacian. J. Diff. Eq. 159.1 (1999): 212-238. 9

[CDG2] M. Cuesta, D. G. De Figueiredo, Jean-Pierre Gossez. A nodal domain property for the p-Laplacian. Comptes Rendus Acad. Sc.-Ser. I-Math. 330 (2000): 669-673. 9

[CT] M. Cuesta, P. Takac. A strong comparison principle for positive solutions of degenerate elliptic equations. Diff. Int. Eq. 13 (2000): 721-746. 9

[DG] F. Della Pietra, N. Gavitone Symmetrization for Neumann anisotropic problems and related questions. Adv. Nonlinear Stud. 12(2), 219?235 (2012). 2

[DG2] F. Della Pietra, N. Gavitone. Faber-Krahn inequality for anisotropic eigenvalue problems with Robin boundary conditions. Potential Anal. 41(2014):1147-1166. 2, 5

[DG3] F. Della Pietra, N. Gavitone. Sharp bounds for the first eigenvalue and the torsional rigidity related to some anisotropic operators. Math. Nachr. 287 (2014): 194-209. 2

[DGP] F. Della Pietra, N. Gavitone, G. Piscitelli, A sharp weighted anisotropic Poincaré inequality for convex domains, preprint. 2

[ET] L. Esposito and C. Trombetti. Convex symmetrization and Pólya-Szegö inequality. Nonlinear Anal. TMA, 56 (2004):43-62. 6

[FV] A. Ferone, R. Volpicelli. Convex rearrangement: equality cases in the Pólya-Szegö inequality. Calc. Var. PDE, 21 (2004):259-272. 6

[F] G. Franzina. Existence, uniqueness, optimization and stability for low eigenvalues of some nonlinear operators. PhD thesis. 2, 6, 9

[JL] P. Juutinen, P. Lindqvist. On the higher eigenvalues for the $\infty$-eigenvalue problem. Calc. Var. 23 (2005): 169-192. 3

[JLM] P. Juutinen, P. Lindqvist and J. Manfredi. The $\infty$-eigenvalue problem. Arch. Rat. Mech. Anal. 148 (1999): 89-105. 2, 11, 12

[KN] B. Kawohl, M. Novaga. The p-Laplace eigenvalue problem as $p \rightarrow 1$ and Cheeger sets in a Finsler metric. J. Convex. Anal. 15 (2008):623-634. 2

[LU] O. Ladyzhenskaya, N. Ural'tseva. Linear and quasilinear elliptic equations, Academic Press, New York, 1968. 8

[L] P. Lindqvist, On the equation div $\left(|\nabla u|^{p-2} \nabla u\right)+\lambda|u|^{p-2} u=0$. Proc. Amer. Math. Soc. 109 (1990), 157-164. 5

[L1] P. Lindqvist. On nonlinear Rayleigh quotient. Potential Anal. 2 (1993): 199-218. 5

[L2] P. Lindqvist. A nonlinear eigenvalue problem. Topics in mathematical analysis 3 (2008): 175-203. 2, 6,7

[P] G. Piscitelli. A nonlocal anisotropic eigenvalue problem. Diff. Int. Eq. 29 (2016), 1001-1020. 2

[WX] G. Wang, C. Xia. An optimal anisotropic Poincaré inequality for convex domains. Pacific J. Math. 258 (2012):305-326 2 